\section{ACCESSIBILITY OF CHILD HEALTH CARE SERVICES DURING A NATIONAL LOCK-DOWN: A PARENTAL SURVEY}

Taiwo Ladapo, Sanjay Wazir Pandita, Abiodun Adu. UK

\subsection{6/bmjpo-2021-RCPCH.118}

Background The morbidity and mortality from the novel coronavirus 19 are less pronounced in children compared with adults. It's impact on the health care services can however indirectly impact the health care delivery to children including accessibility.

Objectives The objective of this survey therefore was to obtain feedback from parents about how they accessed health care for their unwell children during the peak of the COVID 19 pandemic and challenges faced in the process.

Methods All parents attending the paediatric wards and assessment units of a district hospital in England with their children were prospectively interviewed over a 2 -month period using a self-administered questionnaire. Information obtained included health care service accessed during the national lock-down if needed, time to obtain care, mode of consultation, satisfaction with treatment and any deterrents to seeking health care in the hospital. Data was analysed using Microsoft excel and presented using descriptive statistics.

Results We surveyed 103 parents of whom 49(47.6\%) sought healthcare for their children from 1st April to 30th June 2020. There were 31 males and 18 females (M: F, 1: 0.58 ). Number of children seeking health care monthly were 12(24.55\%), 22(44.9\%) and 15(30.6\%) in April, May and June respectively. Source of health care was as follows: General Practitioners 25(51\%); National Health Service 111 helpline 15(30.6\%); Hospital paediatric services 8(16.3\%). One parent was unable to access vaccination services. Time to obtain help was $<1 \mathrm{hr}$ in $20,1-2$ hours in 11 and $>2 \mathrm{hrs}$ in 16 with the greatest delays from GP and 111 services. There was delayed diagnosis of a ruptured appendix in 1 patient which was however successfully treated. The predominant form of consultation was via telephone $(83 \%)$ while video and in person consultations constituted $13 \%$ and 4\% respectively. Thirty-two (64\%) parents expressed satisfaction with treatment received, $8(16 \%)$ were neither satisfied nor dissatisfied while 9 (18.4\%) expressed dissatisfaction. Twelve (24.5\%) parents who would ordinarily have sought hospital care did not for the following reasons: fear of the virus (6), stay at home orders (2), uncertainty about hospital services (1), reluctance to burden the hospital services (1), no reason given (2). However of parents who did not seek hospital care, $83.3 \%$ were satisfied with the treatment received.

Conclusions Paediatric health care remained accessible by most parents during the National lockdown with some expected delays. There is no evidence that any critically ill children were missed although impact on child safeguarding issues could not be looked into. Majority of consultations were virtual and we conclude that strengthening of newly introduced virtual consultations may reduce burden on hospital services during such lock-downs. The public need to be made aware about continuity of hospital services and accessing these for any acutely unwell child needing urgent attention. A larger study over a period of time may be needed to look into the impact of virtual consultation on possible missed or delayed diagnosis.

\section{USE OF PARACETAMOL FOR CLOSURE OF PATENT DUCTUS ARTERIOSUS IN PREMATURE INFANTS IN A LEVEL 3 NICU}

Lizanne Noronha, Anusha Arasu, Joanne Crook. UK

\subsection{6/bmjpo-2021-RCPCH.119}

Background Paracetamol (both IV and PO) has been shown to be effective at closing haemo-dynamically significant Patent Ductus Arteriosus (PDA) in preterm infants. Currently, Ibuprofen is the only licensed medical treatment for PDA in neonates but Paracetamol has been suggested as a second line agent where Ibuprofen therapy has failed or is contraindicated. The aim of this project was to review the use of Paracetamol for the medical management of PDA in a tertiary neonatal unit in London. Patients received IV paracetamol in accordance with the West of Scotland guideline.

Objectives

1. To determine the number of infants treated with intravenous paracetamol for closure of PDA over a 2 year period and determine indication for paracetamol treatment rather than Ibuprofen

2. To determine if any infants suffered adverse effects or needed dose adjustment due to high paracetamol levels. Levels were measured before the $3 \mathrm{rd}$ dose of treatment

3. To determine the number of infants with successful closure of PDA following treatment with intravenous paracetamol

Methods A retrospective analysis was carried out using the online national neonatal database (BADGER). Infants were selected using the following search criteria

- Born between $1 / 3 / 2018$ and $31 / 3 / 2020$ and admitted to NICU

- Diagnosis of PDA confirmed on echo

- Treatment with at least one course of Paracetamol for medical management of PDA

Results 23 babies met the inclusion criteria. Male to female ratio was $11: 12$, gestational age range was $23+1-29+2$ weeks (median 24+6) and birth weight $430 \mathrm{~g}-1514 \mathrm{~g}$ (median $728 \mathrm{~g}$ ). The main reason for medical treatment was ventilator dependence $(22 / 23)$.

14/23 (61\%) were treated with Paracetamol first line, due to contraindications to Ibuprofen

\begin{tabular}{ll} 
Abstract 220 Table 1 & \\
\hline Contraindication to Ibuprofen & Number \\
\hline Deranged renal function & 5 \\
Necrotising enterocolitis & 4 \\
Intraventricular haemorrhage & 2 \\
Deranged clotting & 1 \\
Pulmonary haemorrhage & 1 \\
Not documented & 1 \\
\hline
\end{tabular}

9/23 (39\%) received Paracetamol as a second line treatment, following failed treatment with Ibuprofen

No adverse effects were reported, and no babies required dose adjustment due to high Paracetamol levels, all levels were below the toxic threshold $(25 \mathrm{mg} / \mathrm{L})$ 
Successful closure of PDA was seen in 18/23 (83\%) patients after the following treatment regimens with Paracetamol:

\begin{tabular}{ll} 
Abstract 220 Table 2 & \\
\hline Outcome & Number of patients \\
\hline 1 course Paracetamol & 10 \\
2 courses Paracetamol & 2 \\
1 course Ibuprofen +1 course Paracetamol & 5 \\
2 courses Ibuprofen +1 course paracetamol & 1 \\
\hline
\end{tabular}

Of the remaining 5 patients, outcomes were as follows:

\begin{tabular}{ll} 
Abstract 220 Table 3 & Number of \\
Outcome & 3 \\
\hline $\begin{array}{l}\text { Successful closure of PDA with } 1 \text { course Ibuprofen following } 1 \\
\text { failed course Paracetamol }\end{array}$ \\
$\begin{array}{l}1 \text { failed course of paracetamol, required surgical ligation } \\
2 \text { failed courses paracetamol, } 1 \text { failed course Ibuprofen, required } \\
\text { surgical ligation }\end{array}$ & 1 \\
\hline
\end{tabular}

Conclusions Paracetamol appears to be a safe and effective medical treatment for haemo-dynamically significant PDA in preterm neonates who either have contra-indications to Ibuprofen or where Ibuprofen therapy has failed. The risk of toxicity was low $(0 \%$ in this study) and the closure rate of PDA following treatment was high (83\%). Further studies are needed to determine clinical effectiveness in larger sample sizes and to agree a consensus on how many courses of medical management are appropriate before referring for surgical ligation.

\section{10-YEAR REVIEW OF PATHOGENS AND THEIR ANTIMICROBIAL RESISTANCE PATTERNS AT A TERTIARY NEONATAL INTENSIVE CARE UNIT. DO WE HAVE THE RIGHT ANTIBIOTIC POLICIES?}

Monica Arend-Trujillo, Manjusha Narayanan, Isha Rizal, Naveen Athiraman. UK

\subsection{6/bmjpo-2021-RCPCH.120}

Background The presentation of neonatal sepsis is typically non-specific, and empirical antimicrobial treatment is generally commenced before a causative organism is identified. Using surveillance systems to identify pathogens and their antimicrobial resistance patterns, helps to inform clinicians of what isolates to target and assists them in using antibiotics judiciously. Local antibiotic guideline First line antibiotics:

- Penicillin + Gentamicin.

- Second line: Amoxicillin + Gentamicin + Flucloxacillin.

- Second line where CoNS identified or very likely (e.g., preterm with long line but not systemically 'sick'): Vancomycin

- Third line: Vancomycin and Ceftazidime

\section{Objectives}

- To compile all positive cultures from blood, cerebrospinal fluid and respiratory secretions in a 10 year period

- To identify common causative pathogens in the neonatal intensive care unit.

- To detect susceptibility and resistant patterns to antibiotics recommended in local guideline

Methods Retrospective analysis of data from the microbiology department between 1st April 2010 and 31st March 2020.

Data included all positive blood, cerebrospinal fluid, and respiratory secretions cultures, together with their antimicrobial susceptibility and resistance profile

Results A total of 1642 positive cultures were identified in the 10 -year period, from which $68.4 \%$ came from respiratory secretions, $28.9 \%$ from blood and $2.7 \%$ from cerebrospinal fluid samples.

The five most prevalent organisms in all combined cultures were Coagulase negative staphylococci (CoNS), Staphylococcus aureus, Escherichia coli, Enterobacter species and Klebsiella pneumoniae.

CoNS was the most prevalent organism in blood and cerebrospinal fluid cultures, at $56 \%$ and $34.88 \%$ respectively. GBS was always susceptible to Penicillin and E. coli was resistant to Gentamicin in $5 \%$ of cases.

CoNS was $100 \%$ susceptible to Vancomycin and Linezolid but mostly resistant to Gentamicin and Flucloxacillin.

Conclusions Similar pathogens were seen to national data from other NICUs. No significant difference was seen for most prevalent pathogens in a 10 -year period.

First line antibiotics in our local guideline provided good coverage for early onset sepsis organisms.

Second line antibiotics did not cover CoNS, for patients without central line in situ. However, they provided good cover for all other prevalent pathogens involved in late onset sepsis.

A step up from third line antibiotics is considered, when resistant organisms are suspected or grown, in discussion with the microbiologist.

\section{ACHIEVING SGD 3 GOALS WITH GENETICS, INTERNET TECHNOLOGY AND DATA-DRIVEN IMPROVEMENT}

Liesbeth Siderius, Elmas Citak, Rob Braamburg, Sanath Lamabadururiya, Anton Heys, Marc de Graauw. Netherlands

\subsection{6/bmjpo-2021-RCPCH.121}

Background Some 93 million children under 15 years of age live with a moderate or severe disability (WHO, 2015). Disabilities, such as hearing deficit, cerebral palsy, developmental disabilities, turn out to have genetic causes. In 2019 the WHO launched a classification of digital health interventions v1.0 ${ }^{1}$ : Shared language to describe the uses of digital technology for health. In lower and middle-income countries the use of electronic data in medical care is still on the verge of development. Awareness of available genetic tests could be improved.

Objectives International codes and classification are designed to support data harmonization and data exchange. We questioned how new techniques can promote healthy lives and well-being.

Methods Most states provide maternal and child health handbooks (WHO) containing essential information about 